\title{
On The Edge-balance Index Sets of the Power Circle Nested Graph $C_{m^{m}} \times P_{m}(m \equiv 2(\bmod 3))$
}

\author{
Yanjiao Qin \\ Henan Polytechnic University, Jiaozuo, China \\ Email: qinyanjiao622@163.com \\ Yuge Zheng \\ Henan Polytechnic University, Jiaozuo, China \\ Email: zhengyuge@hpu.edu.cn
}

\begin{abstract}
Based on the equal-cycle nested graph, the powercycle nested graph is brought forward. In this paper, we research on the largest edge-balance index of the graph $C_{7_{m}} \times P_{m_{7}}(m \equiv 2(\bmod 3)) \quad(m \geq 5) \quad$ by the methods and techniques of graph theory and combinatorial mathematics, and solve formula proof and graphic tectonic methods.
\end{abstract}

Index Terms-Edge-friendly labeling, Edge-balanced index set, Graph $C_{7_{m}} \times P_{m_{7}}$, the nested-cycle graph with claw.

\section{INTRODUCTION}

Graph labeling problem starts from A. Rosa's famous beautiful tree conjecture in 1966. Balance index sets are an important branch of graph labeling problem. Boolean index [1] set of graphs is that makes the vertex sets and the edge sets of graphs through the mapping function with $Z_{2}$, to study the characteristics, inherent characteristics of graphs, the structure and design techniques, and to complete the theoretical derivation of the index sets of graphs and formula proof. Balance index set [2] is an important branch of Boolean index sets, edge-balance index set is one of important issues of balance index sets. Its theory can be applied to information engineering, communication network, computer science, economic management, medicine, etc.

Before the years 2006, people mainly studied the edgebalance index of the finite graph. Since 2007, Yuge Zheng with her students has been committed to the research about the edge-balanced index sets of the graphs. In 2008-2009, Juan Lu has completely solved the edgebalance index sets of the series of the infinite chain graph. In 2009, Yurong Ji and M. C. Kong with his partners used the different methods to solve the edge-balanced index sets of the complete graph, respectively in reference [3] [4]. In 2010-2012, Professor Zheng told Ying Wang and Jingjing Yao to completely solve the edge-balance index sets of the equal-cycle nested graph, for details, please refer to [5] [7]. In 2011-2012, elder sister Hongjuan Tian, Yanming Chang and Qingwen Zhang started to research infinite power-cycle nested graph in reference [6], and completed for $n=2,3,4,6,8$. The larger the value of $\mathrm{n}$ is, the more difficult the research of the graph design of odd $n$ is, using the innovation of this mosaic design and the method of the foundation drawing validation. At the same time, using the foundation drawing and toothed nested-cycle graph, we completed the labeling design of the edge-balance index sets of $C_{7^{m}} \times P_{m_{7}}$ graphs and formula derivation.

The rest of this article is organized as follows. In the second part, we give the definitions which will use in this paper. In section 3 we summarize the main results and give a brief overview of the key ideas of their proofs. The main theorem is presented in the fourth section. In the fifth part of the paper, we draw the conclusion.

\section{PRELIMINARY NOTES}

In graph $G$, the edge set of labeled 0 or 1 is recorded as $E(0)$ or $E(1)$, using $e(0), e(1)$ to present the number of $E(0), E(1)$. The vertex set of labeled 0or 1 is recorded as $\mathrm{V}(0)$ or $\mathrm{V}(1)$, using $\mathrm{v}(0), \mathrm{v}(1)$ to present the number of $\mathrm{V}(0), \mathrm{V}(1)$.

In graph $G$, let $f: E(G) \rightarrow Z_{2}$ is an edge labeling function, That is to say, $\forall e \in E(G), f(e)=0$ or 1 . According to the edge labeling $f$, we define an associated partial vertex labeling $f^{+}: V(\mathrm{G}) \rightarrow \mathrm{Z}_{2}$, as follow: $\quad f^{+}(x)=\left\{\begin{array}{c}0, e_{x}(0)>\mathrm{e}_{x}(1) \\ 1, e_{x}(1)>e_{x}(0) \\ \text { unlabled }, e_{x}(0)=e_{x}(1)\end{array}\right.$, where $e_{v}(0)$ is the cardinality of the set $\{e \in E(G): v(e)=0\}, e_{v}(1)$ is the cardinality of the set $\{e \in E(G): v(e)=1\}$.

Definition 1 An edge labeling $f: E(G) \rightarrow\{0,1\}$ of the $\operatorname{graph} G$ is said to be edge-friendly if $\left|e_{f}(0)-e_{f}(1)\right| \leq 1$.

Definition 2 If there is an edge-friendly labeling in a graph, $\left\{\left|v_{f}(0)-v_{f}(1)\right|: f\right.$ is an edge friendly labeling of $G\}$ is called on edge-balance index of $G$ and denoted by $E B I(G)$. Specifically, the maximum of edge-balance index denoted by $\max \{E B I(G)\}$. 
Definition $3 P_{m_{7}}$ represents that every road contains $\mathrm{m}$ points, and that there are 7 branches at any points except the terminal point of each road.

Definition $4 C_{7^{m}}$ shows the graph contains $m$ cycles, the cycles are denoted by the first cycle, the second cycle, the third cycle, $\cdots \cdots$, the m-th cycle from the inner cycle to the outer. And there are $7^{i}$ vertices on the i-th cycle.

Definition 5 The nested graph with power-cycle is the embedded graph including $C_{7^{m}}$ and $P_{m_{7}}$, denoted by $C_{7^{m}} \times P_{m_{7}}$.

Example. Fig. 1 illustrates two graphs of $C_{7^{m}} \times P_{m_{7}}$

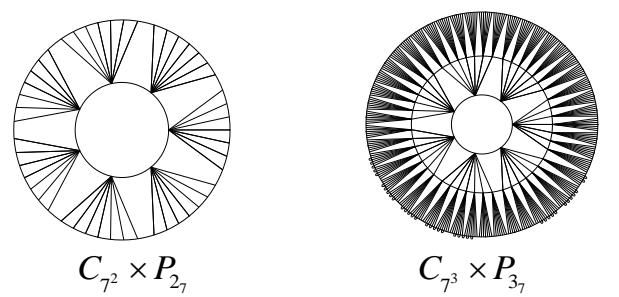

Fig. 1. two graphs of $C_{7_{m}} \times P_{m_{7}}$

For convenience, we will have the following label for the nested network graph: The vertices of the most inner circle in clockwise order are labeled as follows: $(1)_{1},(1)_{2}, \cdots,(1)_{n-1},(1)_{n}$; Similarly, from inside to outside the vertices of the most outside circle in clockwise are labeled as follows: $(m)_{1},(m)_{2},(m)_{3},(m)_{4}, \cdots,(m)_{n^{m}-1},(m)_{n^{m}}$. Among them, symbols $(j)_{i}$ says the $i-t h$ vertex in the $j-t h$ circle. The paths begin with the vertex $(1)_{1}$, as follows:

$$
\begin{aligned}
& (1)_{1} \rightarrow(2)_{1} \rightarrow(3)_{1} \rightarrow \cdots \rightarrow(m-1)_{1} \rightarrow(m)_{1} ; \\
& (1)_{1} \rightarrow(2)_{1} \rightarrow(3)_{1} \rightarrow \cdots \rightarrow(m-1)_{1} \rightarrow(m)_{2} ; \\
& \cdots \cdots \\
& (1)_{1} \rightarrow(2)_{1} \rightarrow(3)_{1} \rightarrow \cdots \rightarrow(m-1)_{1} \rightarrow(m)_{m} ; \\
& (1)_{1} \rightarrow(2)_{1} \rightarrow(3)_{1} \rightarrow \cdots \rightarrow(m-1)_{1} \rightarrow(m)_{m+1} ; \\
& \cdots \cdots \\
& (1)_{1} \rightarrow(2)_{n^{1}} \rightarrow(3)_{n^{2}} \rightarrow \cdots \rightarrow(m-1)_{n^{m-2}} \rightarrow(m)_{n^{m-1}-1} ; \\
& (1)_{1} \rightarrow(2)_{n^{1}} \rightarrow(3)_{n^{2}} \rightarrow \cdots \rightarrow(m-1)_{n^{m-2}} \rightarrow(m)_{n^{m-1}} ;
\end{aligned}
$$

similarly, the paths begin with the vertex $(1)_{n}$, as follows:

$$
\begin{aligned}
& (1)_{n} \rightarrow(2)_{n^{2}-n+1} \rightarrow(3)_{n^{3}-n^{2}+1} \rightarrow \cdots \rightarrow(m-1)_{n^{m-1}-n^{m-2}+1} \rightarrow(m)_{n^{m}-n^{m-1}+1} \\
& (1)_{n} \rightarrow(2)_{n^{2}-n+1} \rightarrow(3)_{n^{3}-n^{2}+1} \rightarrow \cdots \rightarrow(m-1)_{n^{m-1}-n^{m-2}+1} \rightarrow(m)_{n^{m}-n^{m-1}+2} \\
& \ldots \ldots \\
& (1)_{n} \rightarrow(2)_{n^{2}-n+1} \rightarrow(3)_{n^{3}-n^{2}+1} \rightarrow \cdots \rightarrow(m-1)_{n^{m-1}-n^{m-2}+1} \rightarrow(m)_{n^{m}-n^{m-1}+n} \\
& (1)_{n} \rightarrow(2)_{n^{2}-n+1} \rightarrow(3)_{n^{3}-n^{2}+1} \rightarrow \cdots \rightarrow(m-1)_{n^{m-1}-n^{m-2}+1} \rightarrow(m)_{n^{m}-n^{m-1}+n+1} \\
& \ldots \ldots \\
& (1)_{n} \rightarrow(2)_{n^{2}} \rightarrow(3)_{n^{3}} \rightarrow \cdots \rightarrow(m-1)_{n^{m-1}} \rightarrow(m)_{n^{m}-1} ; \\
& (1)_{n} \rightarrow(2)_{n^{2}} \rightarrow(3)_{n^{3}} \rightarrow \cdots \rightarrow(m-1)_{n^{m-1}} \rightarrow(m)_{n^{m}} ;
\end{aligned}
$$

The above says that the $C_{n^{m}} \times P_{m_{n}}$ have $n^{m}$ paths.
Definition 6 A 1-vertex $x$ is considered to be saturated, if the $n$ edges linked to $x$ satisfy $e_{x}(1)=e_{x}(0)+1$, when $\mathrm{n}$ is an odd number; $e_{x}(1)=e_{x}(0)+2$, when $\mathrm{n}$ is an even number ; otherwise, the 1-vertex $x$ is unsaturated. A 0 -vertex $x$ is considered to be saturated, if the $n$ edges linked to $x$ are all 0 -edges; otherwise, the 0 -vertex $x$ is unsaturated. A vertex $x$ is considered to be empty, when the point $x$ satisfy $e_{x}(1)=e_{x}(0)$.

Under the definition of power-cycle nested graph, in order to more convenient to figure symbols function. According to the recursive nature of power-cycle nested graph, given the concept of the clawed nested-cycle subgraph.

Definition 7 In the power-cycle nested graph, the induced sub-graph of the vertices, which the ray paths through the points with the vertices on the $k(t-1)+i$ cycle as starting points and the vertices on the $k t+i$ cycle as the terminal points, denoted by $V_{t}^{\prime}$. And the graph $V_{t}^{\prime}$ subtract the edge on $k(t-1)+i$ is thought as the clawed nested-cycle sub-graph $V_{t}\left(t=1,2, \cdots, \frac{m-i}{k}\right)$.

Specifically, $\mathrm{k}$ be decided by the $\mathrm{m}$ classification. In the graph $C_{7^{m}} \times P_{m_{7}}(m \geq 5)$, refers to all the clawed nested-cycle sub-graphs are the clawed 3 nested-cycle sub-graph.

Under the definition of the clawed nested-cycle subgraph, in order to more convenient to figure symbols function. According to the dual nature of power-cycle nested graph, given the concept of the fan-shaped subgraph.

Definition 8 For a given the clawed nested-cycle subgraph $V_{t}\left(t=1,2, \cdots, \frac{m-i}{k}\right)$, based on the starting points of the ray paths, $V_{t}\left(t=1,2, \cdots, \frac{m-i}{k}\right)$ is divide from the middle the fan graphs, if the starting point in the $\alpha$ circle, it can be divided into $k^{\alpha}$ sector fan-shaped graphs, denoted by $H_{0}, H_{1}, \cdots, H_{K^{\alpha}}$.

Specifically, every the fan sub-graph of the single point does not include its own point.

According to the definition of the clawed nested-cycle nested sub-graph, all of the clawed nested-cycle nested sub-graph $V_{t}\left(t=1,2, \cdots, \frac{m-i}{k}\right)(i=5,6,7)$ are 3 nestedcycle nested sub-graph in the $C_{7^{m}} \times P_{m_{7}}(m \geq 5)$. So if given the clawed nested-cycle sub-graph starting point in the $\alpha$ circle, the corresponding the clawed nested-cycle sub-graph can be divided into $7^{\alpha}$ sector fan-shaped graphs.

In order to more convenient to figure symbols function, we introduce the concept of divisibility for power-cycle nested graph, it is obvious that 
$C_{7^{m}} \times P_{m_{7}}=C_{7^{K}} \times P_{K_{7}} \cup\left(\bigcup_{t=0}^{\frac{m-2}{3}} V_{t}\right)$, according to the 3 circles of the $t$ nested-cycle nested sub-graph $V_{t}$, from the side to the out, in turn, for the $\alpha$ circle, the $\alpha+1$ circle, the $\alpha+2$ circle; $V_{t}$ will be average divided into $7^{3 t+2}$ sector fan-shaped sub-graph $H_{0}$, ensure that each $H_{0}$ has the $7^{i}$ point and the $7^{i}$ edge on the $i$ circle , according to the clockwise order the points on the $i$ circle denoted by $(i)_{1},(i)_{2}, \cdots,(i)_{7^{i}}$.

For the edge-friendly labeling of the graph $C_{7^{m}} \times P_{m_{7}}(m \geq 5)$. We discuss the edge-balance index set of this graph from the following three parts: $m \equiv 0(\bmod 3), m \equiv 1(\bmod 3), m \equiv 2(\bmod 3)$. But, in this paper, we only research it when $m \equiv 2(\bmod 3)$.

\section{LEMMAS AND PROOF}

Lemma 1 In the graph $C_{7^{m}} \times P_{m_{7}}$, for the fan subgraph $H_{0}$ of the single point, $\max \left\{E B I H_{0}\right\}=323$.

Proof: Because each part in the fan sub-graph of the single point have equal features, here we only give label $H_{0}$, the others label as well as itself.

There are 798 edges in the fan sub-graph $H_{0}$ of the single point, because of $|e(0)-e(1)| \leq 1$, there are 3990 edges. To put it simply, we only mark the 0-edges of the fan sub-graph $H_{0}$ of the single point, the left edges are 1edges.

The four edges are all 0-edges except $(\alpha)_{6}(\alpha)_{7},(\alpha)_{5}(\alpha)_{6},(\alpha)_{4}(\alpha)_{5}$ in the $\alpha$ circle, and the $7^{2}$ edges are all 0 -edges in the $\alpha+1$ circle. The edges linked to the vertices $(\alpha)_{i}(1 \leq i \leq 3)$ are all 0 -edges in the $\alpha$ circle; The edges linked to the vertices $(\alpha+1)_{i}(1 \leq i \leq 24,29 \leq i \leq 32,39 \leq i \leq 42,47 \leq i \leq 49)$ are all 0 edges in the $\alpha+1$ circle; The 0 -edges between the $\alpha$ circle and the $\alpha+1$ circle: $(\alpha)_{i}(\alpha+1)_{j}$ $(i=4,22 \leq j \leq 24)(i=5,29 \leq j \leq 32)(i=6,39 \leq j \leq 42)$ $(i=7,47 \leq j \leq 49)$; The 0 -edges between the $\alpha+1$ circle and the $\alpha+2$ circle: $(\alpha+1)_{i}(\alpha+2)_{j}$ $(j=7(i-1)+k, i=25+2 l, 0 \leq l \leq 1,4 \leq l \leq 6,9 \leq l \leq 10, k=1,2)$ $(j=7 i+k, i=26+2 l, 0 \leq l \leq 1,4 \leq l \leq 6,9 \leq l \leq 10, k=-1,0) \quad$; In the $\alpha+2$ circle the edges are all 0 -edges: $(\alpha+2)_{i}(\alpha+2)_{i+1} \quad(i=170+7 k+j, k=2 l, 0 \leq l \leq 1,4 \leq l \leq 6,9 \leq l \leq 10, j=1,3,5,7,9)$

Then $v(0)=38,|v(1)-v(0)|=|v-2 v(0)|=323$.

We can get the edge-friendly labeling of the fan subgraph $H_{0}$ of the single point by calculation, then not conclude the vertices are not defined and unsaturated. When we change the 0 -vertex into 1 -vertex or undefined vertex, we must take out five 0 -edges. Thus, wherever we put the five 0 -edges, the number of the 0 -vertex will increase, meanwhile the number of the 1-vertex will not increase. So 323 is the maximum edge-balance index.

$$
\text { So } \max \left\{E B I H_{0}\right\}=323 \text {. }
$$

Lemma 2 For the power-cycle nested graph $C_{7^{m}} \times P_{m_{7}}$, when $m=5, \max \left\{E B I\left(C_{7^{5}} \times P_{5_{7}}\right)\right\}=15873$.

Proof: There are 39207 edges in the power-cycle nested graph $C_{7^{5}} \times P_{5_{7}}$, because of $|e(0)-e(1)| \leq 1$, there are 19603 0-edges. To put it simply, we only mark the 0edges of the graph, the left edges are 1-edges.

The edges are all 0 -edges in the 2 circle and in the 3 circle and in the 4 circle. In the 1 circle the edges are all 0-edges: $(1)_{2}(1)_{3},(1)_{4}(1)_{5},(1)_{6}(1)_{7},(1)_{7}(1)_{1}$.

First of all, edges in all the paths which set out from the labeled point $(1)_{1}$ : the edges linked to the vertices $(2)_{i}(1 \leq i \leq 3)$ are all 0 -edges in the 2 circle; The edges linked to the vertices $(3)_{i}(1 \leq i \leq 23)(i=33+j, 1 \leq j \leq 4)(i=48,49)$ are all 0 -edges in the 3 circle; The 0 -edges between the 2 circle and the 3 circle:

$$
(2)_{4}(3)_{i}(i=22,23) \quad, \quad(2)_{5}(3)_{i}(i=34,35) \quad, \quad(2)_{6}(3)_{i}(i=36,37) \quad,
$$$$
(2)_{7}(3)_{i}(i=48,49) \text {; }
$$

The edges linked to the vertices

$(4)_{i}(1 \leq i \leq 163)(i=173+7 k+j, k=2 l, 0 \leq l \leq 3, j=1,2,3,4)$ $(i=271+7 k+j, k=2 l, 0 \leq l \leq 3, j=1,2,3,4)(230 \leq i \leq 261)$

$(328 \leq i \leq 343)$ are all 0 -edges in the 4 circle; The 0 -edges between the 3 circle and the 4 circle: (3) ${ }_{i}(4)_{j}(j=7(i-1)+k, i=24+2 l, 0 \leq l \leq 4,7 \leq l \leq 11, k=1,2)$ $(j=7 i+k, i=25+2 l, 0 \leq l \leq 4,7 \leq l \leq 11, k=-1,0) \quad$; The 0 edges between the 4 circle and the 5 circle: $(4)_{i}(5)_{j}$

$(j=7(i-1)+k, i=164+2$ lor $262+2 l, 0 \leq l \leq 4,7 \leq l \leq 11$,

$14 \leq l \leq 18,21 \leq l \leq 25,28 \leq l \leq 32, k=1,2) \quad(j=7 i+k, i=165+2$ lor $262+2 l$, $0 \leq l \leq 4,7 \leq l \leq 11,14 \leq l \leq 18,21 \leq l \leq 25,28 \leq l \leq 32, k=-1,0)$; In the 5 circle the edges are all 0-edges $(5)_{i}(5)_{i+1}$ $(i=1143+7 k+$ jor $1829+7 k+j, k=2 l, 0 \leq l \leq 4,7 \leq l \leq 11$, $14 \leq l \leq 18,21 \leq l \leq 25,28 \leq l \leq 32, j=1,3,5,7,9)$.

The second, edges in all the paths which set out from the labeled point $(1)_{7}$ : the 0 -edges between the 1 circle and the 2 circle: $(1)_{7}(2)_{i}(43 \leq i \leq 49)$; The edges linked to the vertices $(2)_{i}(i=43,44)$ are all 0 -edges in the 2 circle; The edges linked to the vertices (3) $(295 \leq i \leq 308)(i=315,316)(i=329,330,343)$ are all 0 -edges in the 3 circle; The 0 -edges between the 2 circle and the 3 circle: $\quad(2)_{45}(3)_{315},(2)_{46}(3)_{316},(2)_{47}(3)_{329},(2)_{48}(3)_{330},(2)_{49}(3)_{343} ; \quad$ The edges linked to the vertices $(4)_{i}(2059 \leq i \leq 2158)$

$$
\begin{aligned}
& (i=2168+7 k+j, k=2 l, 0 \leq l \leq 2, j=1,2,3,4) \\
& (i=2224+7 k+j o r 2322+7 k+j, k=2 l, 0 \leq l \leq 4, j=1,2,3,4) \\
& (2201 \leq i \leq 2214)(2295 \leq i \leq 2312)(2393 \leq i \leq 2401)
\end{aligned}
$$

are all 0 -edges in the 4 circle; The 0 -edges between the 3 circle and the 4 circle: $(3)_{i}(4){ }_{j}(j=7 i+k, i=310+2 l$, 


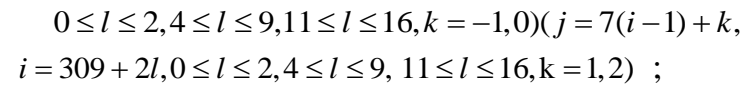

The 0-edges between the 4 circle and the 5 circle: $(4)_{i}(5)_{j}$

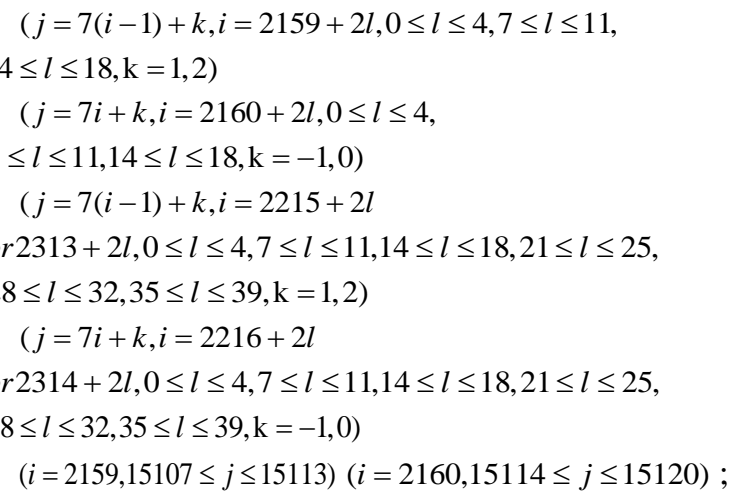

In the 5 circle the edges are all 0-edges: $(5)_{i}(5)_{i+1}(i=15500+7 k+j o r 16186+7 k+j$, $k=2 l, 0 \leq l \leq 4,7 \leq l \leq 11,14 \leq l \leq 18,21 \leq l \leq 25,28 \leq l \leq 32,35 \leq l \leq 39, j=1,3,5,7,9)$ $(i=15134+7 k+j, k=2 l, 0 \leq l \leq 2,5 \leq l \leq 9,12 \leq l \leq 16, j=1,3,5,7,9)$

The last, the label methods of the point $(1)_{i}(2 \leq i \leq 6)$ set out the paths' edge is the same as the point $(1)_{1}$.

Then $v(0)=1867,|v(1)-v(0)|=|v-2 v(0)|=15873$.

In this structure graph, the degree of the vertices is 9 on the first cycle, the degree of the vertices is 10 on the second and third cycle and fourth cycle, the degree of the vertices on the fifth is 3 . In the 1867 0-vertexes, the vertices are all saturated, except the vertices $(4)_{2159},(4)_{2160}$ Therefore, in order to change the labeling of the 0 vertexes, we need to interchange 50 -edges and 51 -edges. It is obvious that the interchanging must bring that the value of $v(0)$ increases or remains unchanged, and the value of $v(1)$ certainly decreases. So the value of $|v(0)-v(1)|$ reduces.

Then the value of $|v(0)-v(1)|$ is maximum in the aforementioned structure graph, so $\max \left\{\operatorname{EBI}\left(C_{7^{5}} \times P_{5_{7}}\right)\right\}=15873$.

Lemma 3 For the power-cycle nested graph $C_{7^{m}} \times P_{m_{7}}$, when $\quad m \equiv 2(\bmod 3)$ and $m \geq 5$ $\max \left\{\operatorname{EBI}\left(C_{7^{m}} \times P_{m_{7}}\right)\right\}=\frac{17 \cdot 7^{m}-5}{18}$.

Proof: There are $\frac{7^{m+1}-28}{3}$ edges in the power-cycle nested graph $C_{7^{m}} \times P_{m_{\eta}}$, because of $|e(0)-e(1)| \leq 1$, there are $\frac{7^{m+1}-31}{6} 0$-edges.

When $m=5$, the formula can be proved by lemma 2 .

When $m \geq 8$, as the foundation graph $C_{7^{5}} \times P_{5_{7}}$ by lemma 1 , denoted by $U_{0}=C_{7^{5}} \times P_{5_{7}}$,

$$
C_{7^{m}} \times P_{m_{\urcorner}}=U_{0} \cup\left(\bigcup_{t=0}^{\frac{m-2}{3}} V_{t}\right)=U_{0} \cup 7^{5} H_{0} \cup 7^{8} H_{0} \cup \cdots \cup 7^{m-3} H_{0} .
$$

Each of the vertices of the outer circle be seen as the starting point to make the graph $H_{0}$ in the figure $C_{7^{m-3}} \times P_{m-3_{7}}$, there are $7^{m-3} H_{0}$. In this graph $C_{7^{m}} \times P_{m_{7}}$,

$$
\begin{aligned}
& v(0)=\left(7^{m-3}+7^{m-6}+\cdots+7^{8}+7^{5}\right) \times 38+1867=\frac{7^{m}-4}{9}, \\
& |v(1)-v(0)|=|v-2 v(0)|=\left|\frac{7^{m+1}-7}{6}-2 \times \frac{7^{m}-4}{9}\right|=\frac{17 \cdot 7^{m}-5}{18} .
\end{aligned}
$$

In this structure graph $C_{7^{m}} \times P_{m_{7}}$, the degree of the vertices is 9 on the first cycle, the degree of the vertices is 3 on $\mathrm{m}$ cycle and all of the others are 10 . All of the 1vertexes are saturated, except the vertices $(4)_{2159},(4)_{2160}$. The edges linked to the other 0 -vertexes are all 0 -edges. Therefore, in order to change the labeling of the 0 vertexes, we need to interchange 50 -edges and 51 -edges. It is obvious that the interchanging must bring that the value of $v(0)$ increases or remains unchanged, and the value of $v(1)$ certainly decreases. So the value of $|v(0)-v(1)|$ reduces.

So for the power-cycle nested graph $C_{7^{m}} \times P_{m_{7}}$, when

$$
m \equiv 2(\bmod 3) m \geq 5, \max \left\{E B I\left(C_{7^{m}} \times P_{m_{7}}\right)\right\}=\frac{17 \cdot 7^{m}-5}{18} .
$$

Specifically, Each of the vertices of the outer circle be seen as the starting point to make the graph $H_{0}$ in the figure $C_{7^{m-3}} \times P_{m-3_{7}}$, the label of each starting point is the same as the boundary point of five laps foundation figure, and it stays the same.

Lemma 4 For the power-cycle nested graph $C_{7^{m}} \times P_{m_{7}}$, when $m=5,\{15872,15871, \cdots, 1,0\} \subset \operatorname{EBI}\left(C_{7^{5}} \times P_{5_{7}}\right)$.

Proof: In the following proof, we use $(2 k-1)_{r}(2 k)_{s} \leftrightarrow(2 k)_{s}(2 k)_{s+1}$ to present that the edge $(2 k-1)_{r}(2 k)_{s}$ is from 0 -edge into1-edge, at the same time the edge $(2 k)_{s}(2 k)_{s+1}$ is from 1-edge into0-edge. Among them, we use $(5)_{i}(6)_{7 i} \leftrightarrow(6)_{7 i}(6)_{7 i+1}$ to present that the edge $(5)_{i}(6)_{7 i}$ is from 1-edge into 0 -edge, at the same time the edge $(6)_{7 i}(6)_{7 i+1}$ is from 0-edge into 1-edge.

First, build an odd index set:

$$
\begin{aligned}
& \text { Step1: }(4)_{i}(5)_{j+7(i-1)} \leftrightarrow(5)_{j+7(i-1)}(5)_{j+7(i-1)+1} \\
& (i=1, \cdots, 163, j=1, \cdots, 4)
\end{aligned}
$$

and

$(5)_{i}(6)_{7 i} \leftrightarrow(6)_{7 i}(6)_{7 i+1}$ $(i=j+7(k-1), k=1, \cdots, 163, j=2, \cdots 5) \quad$, we can get $|v(1)-v(0)|=|v-2 v(0)|=\{15871,15869, \cdots, 14569\}$

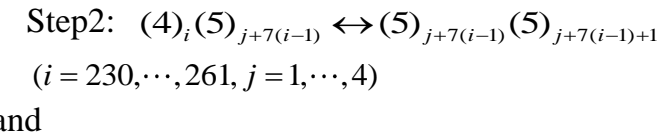


$(5)_{i}(6)_{7 i} \leftrightarrow(6)_{7 i}(6)_{7 i+1}$

$(i=j+7(k-1), k=230, \cdots, 261, j=2, \cdots 5) \quad$, we can get $|v(1)-v(0)|=|v-2 v(0)|=\{14567,14565, \cdots, 14313\}$

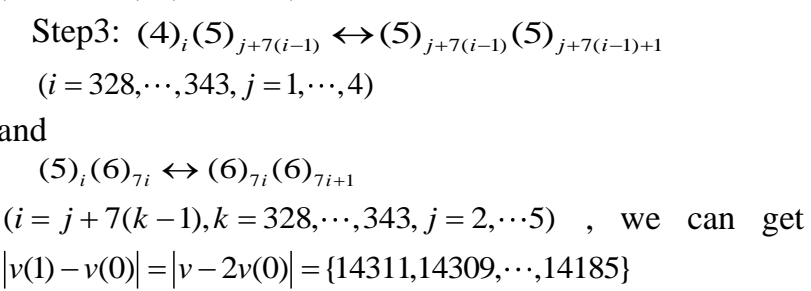

Step4: $(4)_{i}(5)_{j+7(i-1)} \leftrightarrow(5)_{j+7(i-1)}(5)_{j+7(i-1)+1}$

$(i=173+7 n+m, n=2 l, 0 \leq l \leq 3,7 \leq l \leq 10, m=1, \cdots 4$, $j=1, \cdots 4)$

and

$(5)_{i}(6)_{7 i} \leftrightarrow(6)_{7 i}(6)_{7 i+1}(i=j+7(k-1)$,

$k=173+7 n+m, n=2 l, 0 \leq l \leq 3,7 \leq l \leq 10, m=1, \cdots, 4, j=2, \cdots, 5)$, we can get $|v(1)-v(0)|=|v-2 v(0)|=\{14183,14181, \cdots, 13929\}$

Step5: $(4)_{i}(5)_{j+7(i-1)} \leftrightarrow(5)_{j+7(i-1)}(5)_{j+7(i-1)+1}$ $(i=164+2$ lor $262+2 l, 0 \leq l \leq 4,7 \leq l \leq 11,14 \leq l \leq 18,21 \leq l \leq 25$, $28 \leq l \leq 32, j=1,2)$

and

$(5)_{i}(6)_{7 i} \leftrightarrow(6)_{7 i}(6)_{7 i+1}$

$(i=j+7(k-1), k=164+2$ lor $262+2 l, 0 \leq l \leq 4,7 \leq l \leq 11$,

$14 \leq l \leq 18,21 \leq l \leq 25,28 \leq l \leq 32, j=2,3)$, we can get $|v(1)-v(0)|=|v-2 v(0)|=\{13927,13925, \cdots, 13729\}$

Step6: $(4)_{i}(5)_{j+7(i-1)} \leftrightarrow(5)_{j+7(i-1)}(5)_{j+7(i-1)+1}$ $(i=165+2$ lor $263+2 l, 0 \leq l \leq 4,7 \leq l \leq 11,14 \leq l \leq 18,21 \leq l \leq 25$, $28 \leq l \leq 32, j=-1,0$ )

and

$(5)_{i}(6)_{7 i} \leftrightarrow(6)_{7 i}(6)_{7 i+1}$

$(i=j+7 k, k=165+2$ lor $263+2 l, 0 \leq l \leq 4,7 \leq l \leq 11$,

$14 \leq l \leq 18,21 \leq l \leq 25,28 \leq l \leq 32, j=0,1)$, we can get $|v(1)-v(0)|=|v-2 v(0)|=\{13727,13725, \cdots, 13529\}$

Step7: Similar to the first, second, third, fourth, fifth, sixth step transformation, we can obtain these labeled graphs of edge-balance indexes which are $\{13527,13525, \cdots, 1809\}$

Step8: $(4)_{i}(5)_{j+7(i-1)} \leftrightarrow(5)_{j+7(i-1)}(5)_{j+7(i-1)+1}$

$(i=2059, \cdots, 2158, j=1, \cdots, 4)$

and

$(5)_{i}(6)_{7 i} \leftrightarrow(6)_{7 i}(6)_{7 i+1}$

$(i=j+7(k-1), k=2059, \cdots, 2158, j=2, \cdots 5)$, we can get $|v(1)-v(0)|=|v-2 v(0)|=\{1807,1805, \cdots, 1009\}$

Step9: $(4)_{i}(5)_{j+7(i-1)} \leftrightarrow(5)_{j+7(i-1)}(5)_{j+7(i-1)+1}$

$(i=2197, \cdots, 2214,2295, \cdots, 2312,2393, \cdots, 2401, j=1, \cdots, 4)$ and

$(5)_{i}(6)_{7 i} \leftrightarrow(6)_{7 i}(6)_{7 i+1}(i=j+7(k-1)$,

$k=2197, \cdots, 2214,2295, \cdots, 2312,2393, \cdots, 2401, j=1, \cdots, 4)$

we can get $|v(1)-v(0)|=|v-2 v(0)|=\{1007,1005, \cdots, 649\}$

Step10: $(4)_{i}(5)_{j+7(i-1)} \leftrightarrow(5)_{j+7(i-1)}(5)_{j+7(i-1)+1}$ $(i=2168+7 n+m, n=2 l, 0 \leq l \leq 1,4 \leq l \leq 8,11 \leq l \leq 15, m=1, \cdots 4$, $j=1, \cdots 4)$

Copyright (C) 2014 MECS and

$(5)_{i}(6)_{7 i} \leftrightarrow(6)_{7 i}(6)_{7 i+1}(i=j+7(k-1)$,

$k=2168+7 n+m, n=2 l, 0 \leq l \leq 1,4 \leq l \leq 8,11 \leq l \leq 15, m=1, \cdots, 4, j=2, \cdots, 5)$, we can get $|v(1)-v(0)|=|v-2 v(0)|=\{647,645, \cdots, 265\}$

Step11: (4) (5) $_{j+7(i-1)} \leftrightarrow(5)_{j+7(i-1)}(5)_{j+7(i-1)+1}$ $(i=2159+2 l, 0 \leq l \leq 4,7 \leq l \leq 11,14 \leq l \leq 18,21 \leq l \leq 25$,

$28 \leq l \leq 32,35 \leq l \leq 39,42 \leq l \leq 46,49 \leq l \leq 51, j=1,2)$ and $(5)_{i}(6)_{7 i} \leftrightarrow(6)_{7 i}(6)_{7 i+1}(i=j+7(k-1)$, $k=2159+2 l, 0 \leq l \leq 4,7 \leq l \leq 11,14 \leq l \leq 18,21 \leq l \leq 25$, $28 \leq l \leq 32,35 \leq l \leq 39,42 \leq l \leq 46,49 \leq l \leq 51, j=2,3)$, we can get $|v(1)-v(0)|=|v-2 v(0)|=\{263,261, \cdots, 133\}$

Step12: (4) $)_{i}(5)_{j+7(i-1)} \leftrightarrow(5)_{j+7(i-1)}(5)_{j+7(i-1)+1}$ $(i=2160+2 l, 0 \leq l \leq 4,7 \leq l \leq 11,14 \leq l \leq 18,21 \leq l \leq 25$, $28 \leq l \leq 32,35 \leq l \leq 39,42 \leq l \leq 46,49 \leq l \leq 51, j=-1,0)$ and $(5)_{i}(6)_{7 i} \leftrightarrow(6)_{7 i}(6)_{7 i+1}(i=j+7 k$, $k=2160+2 l, 0 \leq l \leq 4,7 \leq l \leq 11,14 \leq l \leq 18,21 \leq l \leq 25$, $28 \leq l \leq 32,35 \leq l \leq 39,42 \leq l \leq 46,49 \leq l \leq 51, j=0,1)$, we can get $|v(1)-v(0)|=|v-2 v(0)|=\{131,129, \cdots, 1\}$

Even index set structures as follows:

Step1: (2) (3) $_{24} \leftrightarrow(3)_{24}(3)_{25}$,

we can get $|v(1)-v(0)|=|v-2 v(0)|=15872$

Step2: (4) (5) $_{j+7(i-1)} \leftrightarrow(5)_{j+7(i-1)}(5)_{j+7(i-1)+1}$ $(i=1, \cdots, 163, j=1, \cdots, 4)$

and

$(5)_{i}(6)_{7 i} \leftrightarrow(6)_{7 i}(6)_{7 i+1}$

$(i=j+7(k-1), k=1, \cdots, 163, j=2, \cdots 5) \quad$, we can get $|v(1)-v(0)|=|v-2 v(0)|=\{15870,15868, \cdots, 14568\}$

Step3: (4) ${ }_{i}(5)_{j+7(i-1)} \leftrightarrow(5)_{j+7(i-1)}(5)_{j+7(i-1)+1}$

$(i=230, \cdots, 261, j=1, \cdots, 4)$

and

$(5)_{i}(6)_{7 i} \leftrightarrow(6)_{7 i}(6)_{7 i+1}$

$(i=j+7(k-1), k=230, \cdots, 261, j=2, \cdots 5) \quad$, we can get $|v(1)-v(0)|=|v-2 v(0)|=\{14566,14564, \cdots, 14312\}$

Step4: $(4)_{i}(5)_{j+7(i-1)} \leftrightarrow(5)_{j+7(i-1)}(5)_{j+7(i-1)+1}$ $(i=328, \cdots, 343, j=1, \cdots, 4)$

and

$(5)_{i}(6)_{7 i} \leftrightarrow(6)_{7 i}(6)_{7 i+1}$ $(i=j+7(k-1), k=328, \cdots, 343, j=2, \cdots 5) \quad$, we can get $|v(1)-v(0)|=|v-2 v(0)|=\{14310,14308, \cdots, 14184\}$

Step5: (4) (5) $_{j+7(i-1)} \leftrightarrow(5)_{j+7(i-1)}(5)_{j+7(i-1)+1}$ $(i=173+7 n+m, n=2 l, 0 \leq l \leq 3,7 \leq l \leq 10, m=1, \cdots 4$, $j=1, \cdots 4)$

and

$(5)_{i}(6)_{7 i} \leftrightarrow(6)_{7 i}(6)_{7 i+1}(i=j+7(k-1)$,

$k=173+7 n+m, n=2 l, 0 \leq l \leq 3,7 \leq l \leq 10, m=1, \cdots, 4, j=2, \cdots, 5)$, we can get $|v(1)-v(0)|=|v-2 v(0)|=\{14182,14180, \cdots, 13928\}$

Step6: (4) ${ }_{i}(5)_{j+7(i-1)} \leftrightarrow(5)_{j+7(i-1)}(5)_{j+7(i-1)+1}$ $(i=164+2$ lor $262+2 l, 0 \leq l \leq 4,7 \leq l \leq 11,14 \leq l \leq 18,21 \leq l \leq 25$, $28 \leq l \leq 32, j=1,2)$ and

I.J. Intelligent Systems and Applications, 2014, 07, 22-28 
$(5)_{i}(6)_{7 i} \leftrightarrow(6)_{7 i}(6)_{7 i+1}$

$(i=j+7(k-1), k=164+2$ lor $262+2 l, 0 \leq l \leq 4,7 \leq l \leq 11$,

$14 \leq l \leq 18,21 \leq l \leq 25,28 \leq l \leq 32, j=2,3)$, we can get $|v(1)-v(0)|=|v-2 v(0)|=\{13926,13924, \cdots, 13728\}$

Step7: (4) $)_{i}(5)_{j+7(i-1)} \leftrightarrow(5)_{j+7(i-1)}(5)_{j+7(i-1)+1}$ $(i=165+2$ lor $263+2 l, 0 \leq l \leq 4,7 \leq l \leq 11,14 \leq l \leq 18,21 \leq l \leq 25$, $28 \leq l \leq 32, j=-1,0$ )

and

$(5)_{i}(6)_{7 i} \leftrightarrow(6)_{7 i}(6)_{7 i+1}$

$(i=j+7 k, k=165+2$ lor $263+2 l, 0 \leq l \leq 4,7 \leq l \leq 11$,

$14 \leq l \leq 18,21 \leq l \leq 25,28 \leq l \leq 32, j=0,1)$, we can get $|v(1)-v(0)|=|v-2 v(0)|=\{13726,13724, \cdots, 13528\}$

Step8: Similar to the first, second, third, fourth, fifth, sixth step transformation, we can obtain these labeled graphs of edge-balance indexes which are $\{13526,13524, \cdots, 1808\}$

Step9: $(4)_{i}(5)_{j+7(i-1)} \leftrightarrow(5)_{j+7(i-1)}(5)_{j+7(i-1)+1}$

$(i=2059, \cdots, 2158, j=1, \cdots, 4)$ and

$(5)_{i}(6)_{7 i} \leftrightarrow(6)_{7 i}(6)_{7 i+1}$

$(i=j+7(k-1), k=2059, \cdots, 2158, j=2, \cdots 5)$, we can get $|v(1)-v(0)|=|v-2 v(0)|=\{1806,1804, \cdots, 1008\}$

Step10: $(4)_{i}(5)_{j+7(i-1)} \leftrightarrow(5)_{j+7(i-1)}(5)_{j+7(i-1)+1}$ $(i=2197, \cdots, 2214,2295, \cdots, 2312,2393, \cdots, 2401, j=1, \cdots, 4)$ and

$(5)_{i}(6)_{7 i} \leftrightarrow(6)_{7 i}(6)_{7 i+1}(i=j+7(k-1)$, $k=2197, \cdots, 2214,2295, \cdots, 2312,2393, \cdots, 2401, j=1, \cdots, 4)$

we can get $|v(1)-v(0)|=|v-2 v(0)|=\{1006,1004, \cdots, 648\}$

Step11: (4) (5) $_{j+7(i-1)} \leftrightarrow(5)_{j+7(i-1)}(5)_{j+7(i-1)+1}$ $(i=2168+7 n+m, n=2 l, 0 \leq l \leq 1,4 \leq l \leq 8,11 \leq l \leq 15, m=1, \cdots 4$, $j=1, \cdots 4)$ and $(5)_{i}(6)_{7 i} \leftrightarrow(6)_{7 i}(6)_{7 i+1}(i=j+7(k-1)$,

$k=2168+7 n+m, n=2 l, 0 \leq l \leq 1,4 \leq l \leq 8,11 \leq l \leq 15, m=1, \cdots, 4, j=2, \cdots, 5)$, we can get $|v(1)-v(0)|=|v-2 v(0)|=\{646,644, \cdots, 264\}$

Step12: $(4)_{i}(5)_{j+7(i-1)} \leftrightarrow(5)_{j+7(i-1)}(5)_{j+7(i-1)+1}$ $(i=2159+2 l, 0 \leq l \leq 4,7 \leq l \leq 11,14 \leq l \leq 18,21 \leq l \leq 25$, $28 \leq l \leq 32,35 \leq l \leq 39,42 \leq l \leq 46,49 \leq l \leq 51, j=1,2)$ and

$(5)_{i}(6)_{7 i} \leftrightarrow(6)_{7 i}(6)_{7 i+1}(i=j+7(k-1)$,

$k=2159+2 l, 0 \leq l \leq 4,7 \leq l \leq 11,14 \leq l \leq 18,21 \leq l \leq 25$,

$28 \leq l \leq 32,35 \leq l \leq 39,42 \leq l \leq 46,49 \leq l \leq 51, j=2,3)$,

we can get $|v(1)-v(0)|=|v-2 v(0)|=\{262,260, \cdots, 132\}$

Step13: (4) (5) $_{j+7(i-1)} \leftrightarrow(5)_{j+7(i-1)}(5)_{j+7(i-1)+1}$

$(i=2160+2 l, 0 \leq l \leq 4,7 \leq l \leq 11,14 \leq l \leq 18,21 \leq l \leq 25$,

$28 \leq l \leq 32,35 \leq l \leq 39,42 \leq l \leq 46,49 \leq l \leq 51, j=-1,0)$ and

$(5)_{i}(6)_{7 i} \leftrightarrow(6)_{7 i}(6)_{7 i+1}(i=j+7 k$,

$k=2160+2 l, 0 \leq l \leq 4,7 \leq l \leq 11,14 \leq l \leq 18,21 \leq l \leq 25$,

$28 \leq l \leq 32,35 \leq l \leq 39,42 \leq l \leq 46,49 \leq l \leq 51, j=0,1)$,

we can get $|v(1)-v(0)|=|v-2 v(0)|=\{130,128, \cdots, 0\}$

In conclusion, we can prove $\{15872,15871, \cdots, 1,0\} \subset E B I\left(C_{7^{5}} \times P_{5_{7}}\right)$.
Lemma 5 In the graph $C_{7^{m}} \times P_{m_{7}}$, for the fan subgraph $H_{0}$ of the single point, $\{322,321, \cdots, 2,1,0\} \subset E B I H_{0}$.

Proof: In the following proof, we use $(2 k-1)_{r}(2 k)_{s} \leftrightarrow(2 k)_{s}(2 k)_{s+1}$ to present that the edge $(2 k-1)_{r}(2 k)_{s}$ is from 0 -edge into1-edge, at the same time the edge $(2 k)_{s}(2 k)_{s+1}$ is from 1-edge into 0-edge. Among them, we use $(\alpha+2)_{i}(\alpha)_{7 i} \leftrightarrow(\alpha)_{7 i}(\alpha)_{7 i+1}$ to present that the edge $(\alpha+2)_{i}(\alpha)_{7 i}$ is from 1-edge into 0-edge, at the same time the edge $(\alpha)_{7 i}(\alpha)_{7 i+1}$ is from 0-edge into 1-edge.

First, build an even index set:

Step1: $(\alpha)_{4}(\alpha+1)_{25} \leftrightarrow(\alpha+1)_{25}(\alpha+1)_{26}$, we can get $|v(1)-v(0)|=|v-2 v(0)|=322$

Step2: $(\alpha+1)_{i}(\alpha+2)_{j+7(i-1)} \leftrightarrow(\alpha+2)_{j+7(i-1)}(\alpha+2)_{j+7(i-1)+1}$ $(i=1, \cdots, 24, j=1, \cdots, 4)$

and

$(\alpha+2)_{i}(\alpha)_{7 i} \leftrightarrow(\alpha)_{7 i}(\alpha)_{7 i+1}$

$(i=j+7(k-1), k=1, \cdots, 24, j=2, \cdots 5)$, we can get $|v(1)-v(0)|=|v-2 v(0)|=\{320,318, \cdots, 130\}$

Step3: $(\alpha+1)_{i}(\alpha+2)_{j+7(i-1)} \leftrightarrow(\alpha+2)_{j+7(i-1)}(\alpha+2)_{j+7(i-1)+1}$

(29 $\leq i \leq 32,39 \leq i \leq 42,47 \leq i \leq 49, j=1, \cdots, 4)$ and

$(\alpha+2)_{i}(\alpha)_{7 i} \leftrightarrow(\alpha)_{7 i}(\alpha)_{7 i+1}$

$(i=j+7(k-1), 29 \leq k \leq 32,39 \leq k \leq 42,47 \leq k \leq 49, j=2, \cdots 5)$, we can get $|v(1)-v(0)|=|v-2 v(0)|=\{128,126, \cdots, 42\}$

Step4: $(\alpha+1)_{i}(\alpha+2)_{j+7(i-1)} \leftrightarrow(\alpha+2)_{j+7(i-1)}(\alpha+2)_{j+7(i-1)+1}$ $(i=25+2 l, 0 \leq l \leq 1,4 \leq l \leq 6, j=1,2)(i=43, j=1)$ and

$(\alpha+2)_{i}(\alpha)_{7 i} \leftrightarrow(\alpha)_{7 i}(\alpha)_{7 i+1}$

$(i=j+7(k-1), k=25+2 l, 0 \leq l \leq 1,4 \leq l \leq 6, j=2,3)$, we can get $|v(1)-v(0)|=|v-2 v(0)|=\{40,38, \cdots, 20\}$

Step5: $(\alpha+1)_{i}(\alpha+2)_{j+7(i-1)} \leftrightarrow(\alpha+2)_{j+7(i-1)}(\alpha+2)_{j+7(i-1)+1}$

$(i=26+2 l, 0 \leq l \leq 1,4 \leq l \leq 6, j=-1,0) \quad$ and $\quad(\alpha+2)_{i}(\alpha)_{7 i} \leftrightarrow(\alpha)_{7 i}(\alpha)_{7 i+1}$ ( $i=j+7 k, k=26+2 l, 0 \leq l \leq 1,4 \leq l \leq 6, j=0,1)$, we can get $|v(1)-v(0)|=|v-2 v(0)|=\{18,16, \cdots, 2,0\}$

Odd index set structures as follows:

Step1: $(\alpha+1)_{i}(\alpha+2)_{j+7(i-1)} \leftrightarrow(\alpha+2)_{j+7(i-1)}(\alpha+2)_{j+7(i-1)+1}$ $(i=1, \cdots, 24, j=1, \cdots, 4)$ and $(\alpha+2)_{i}(\alpha)_{7 i} \leftrightarrow(\alpha)_{7 i}(\alpha)_{7 i+1}$ $(i=j+7(k-1), k=1, \cdots, 24, j=2, \cdots 5)$, we can get $|v(1)-v(0)|=|v-2 v(0)|=\{321,319, \cdots, 131\}$

Step2: $(\alpha+1)_{i}(\alpha+2)_{j+7(i-1)} \leftrightarrow(\alpha+2)_{j+7(i-1)}(\alpha+2)_{j+7(i-1)+1}$

$(29 \leq i \leq 32,39 \leq i \leq 42,47 \leq i \leq 49, j=1, \cdots, 4)$ and

$(\alpha+2)_{i}(\alpha)_{7 i} \leftrightarrow(\alpha)_{7 i}(\alpha)_{7 i+1}$

$(i=j+7(k-1), 29 \leq k \leq 32,39 \leq k \leq 42,47 \leq k \leq 49, j=2, \cdots 5)$, we can get $|v(1)-v(0)|=|v-2 v(0)|=\{129,127, \cdots, 43\}$

Step3: $(\alpha+1)_{i}(\alpha+2)_{j+7(i-1)} \leftrightarrow(\alpha+2)_{j+7(i-1)}(\alpha+2)_{j+7(i-1)+1}$ $(i=25+2 l, 0 \leq l \leq 1,4 \leq l \leq 6, j=1,2)(i=43, j=1)$ and 


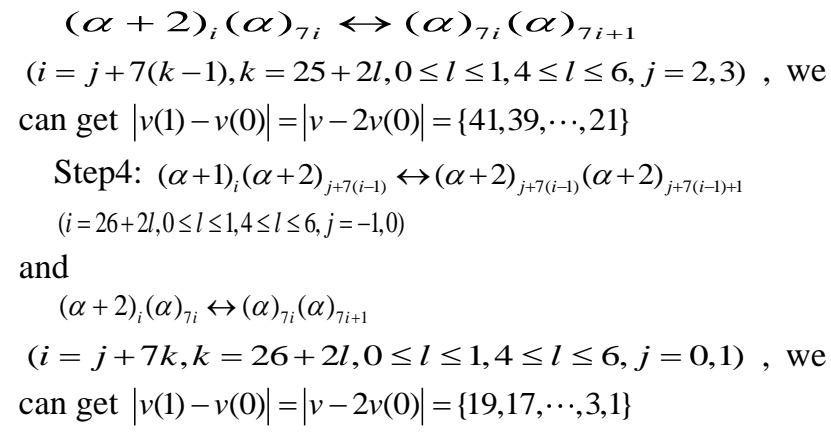

In conclusion, we can prove $\{322,321, \cdots, 1,0\} \subset E B I H_{0}$.

Lemma 6 For the power-cycle nested graph $C_{7^{m}} \times P_{m_{7}}$, when $\quad m \equiv 2(\bmod 3) \quad$ and $\quad m \geq 5$, $\left\{\frac{17 \cdot 7^{m}-23}{18}, \frac{17 \cdot 7^{m}-41}{18}, \cdots, 1,0\right\} \subset \operatorname{EBI}\left(C_{7^{m}} \times P_{m_{7}}\right)$.

Proof: In the lemma 3 , when $m \equiv 2(\bmod 3) m \geq 5$, $\max \left\{\operatorname{EBI}\left(C_{7^{m}} \times P_{m_{7}}\right)\right\}=\frac{17 \cdot 7^{m}-5}{18}$.

Step 1: For the power-cycle nested graph, according to the edge transform method by lemma 5, each of the fan sub-graph $H_{0}$ of the single point index can decrease 323, so $\{322,321, \cdots, 1,0\} \subset E B I H_{0} \quad$. In the graph $C_{7^{m}} \times P_{m}(m \geq 5)$, there are $7^{5}+7^{8}+\cdots+7^{m-3}=\frac{7^{m}-7^{5}}{342}$ the same fan sub-graph of the single point, the edge transform method of the fan sub-graph of single point is the same as lemma 5, then their index can decrease $\frac{7^{m}-7^{5}}{342} \cdot 323=\frac{323\left(7^{m}-7^{5}\right)}{342}$, and $\frac{17 \cdot 7^{m}-5}{18}-\frac{323\left(7^{m}-7^{5}\right)}{342}=15873$. So we can $\frac{7^{m}-7^{5}}{342}$ the fan sub-graph of the single point to conduct edge transform, and we can obtain these labeled graphs of edge-balance indexes which are $\left\{\frac{17 \cdot 7^{m}-23}{18}, \frac{17 \cdot 7^{m}-41}{18}, \cdots, 15873\right\}$.

Step 2: At last, we can make the edge transform in the 5 circle foundation figure. The edge transform method of the 5 lap is the same as lemma 4 , the index can reduce 15873 , so we can obtain these labeled graphs of edgebalance indexes which are $\{15872,15871, \cdots, 1,0\}$.

$$
\begin{aligned}
& \text { In conclusion, we can prove } \\
& \left\{\frac{17 \cdot 7^{m}-23}{18}, \frac{17 \cdot 7^{m}-41}{18}, \cdots, 1,0\right\} \subset E B I\left(C_{7^{m}} \times P_{m_{7}}\right) .
\end{aligned}
$$

\section{THEOREM}

According to lemma 3 and lemma 6, we can get the following theorem.

Theorem 1: For the power-cycle nested graph $C_{7^{m}} \times P_{m_{7}}$, when $m \equiv 2(\bmod 3)$ and $m \geq 5$, $E B I\left(C_{7^{m}} \times P_{m_{7}}\right)=\left\{\frac{17 \cdot 7^{m}-5}{18}, \frac{17 \cdot 7^{m}-23}{18}, \cdots, 1,0\right\}$.

\section{CONCLUSION}

In this paper, in order to study the edge-balanced index sets of the power circle nested graph, we introduced the fan sub-graph of the single point, and showed the proofs of the computational formula, at the same time, we gave the construction of the corresponding graphs.

\section{ACKNOWLEDGMENTS}

This work was financially supported by Applied Mathematics Provincial-level Key Discipline of Henan Province, Operational Research and Cybernetics Key Discipline of Henan Polytechnic University and the Education Department of Henan province science and technology research project (12B110009).

\section{REFERENCES}

[1] M. C. Kong and S. M. Lee, On Edge-Balanced Graphs, Graph Theory, Combinatoric and Algorithms, 1(1995), 711-722.

[2] B. L. Chen, K. C. Huang and S. S. Liu, On Edge-Balanced Multigraphs, Journal of Combinatorial Mathematics and Combinatorial Computing, 42 (2002), 177-185.

[3] M. C. Kong, Y. C. Wang and S. M. Lee, On Edge-Balance Index Set of Some Complete K-partite Graphs, Congressum Numerantium, 196(2009), 71-94.

[4] Yurong Ji and Yuge Zheng, On Edge-Balance Index Sets of the Complete Graphs, 2010 the $2^{\text {nd }}$ IEEE International Conference on Information Management and Engineering, pp. 309-311.

[5] Ying Wang, Yuge Zheng, C.Adiga and A. S. Shrikanth, On the Edge-Balance Index Sets of $\mathrm{N}$ Cycles Three Nested Graph $(n \equiv 0,1,2(\bmod 6))$, Advanced Studied in Contemporary Mathematics, 21(1) (2011), 85-93.

[6] Hongjuan Tian and Yuge Zheng, On the Edge-Balance Index Sets of the Network Graph, Communications in Computer and Information Science, 215(2011), 367-372.

[7] Yuge Zheng and Jingjing Yao, On the Edge-Balance Index Sets of the Equal-cycle nested Graph(1), Journal of Shanghai jiao tong university, 47(7) (2013),1160-1163.

\section{Authors' Profiles}

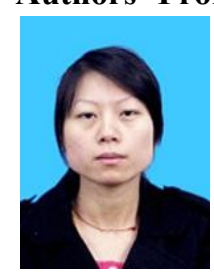

Yanjiao Qin: Student for Master degree for Applied Mathematics in Henan Polytechnic University, major in graph theory and combinatorial mathematics.

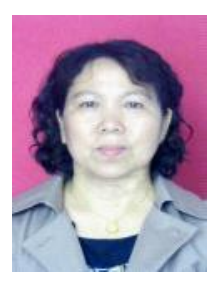

Yuge Zheng: Professor of Henan Polytechnic University, interested in Graph theory and Combinatorial mathematics. 